\title{
Analysis of multipath interference in three-slit experiments
}

\author{
Hans De Raedt, ${ }^{1, *}$ Kristel Michielsen, ${ }^{2, \dagger}$ and Karl Hess ${ }^{3, \ddagger}$ \\ ${ }^{1}$ Department of Applied Physics, Zernike Institute for Advanced Materials, University of Groningen, \\ Nijenborgh 4, NL-9747 AG Groningen, The Netherlands \\ ${ }^{2}$ Institute for Advanced Simulation, Jülich Supercomputing Centre, Forschungszentrum Jülich, D-52425 Jülich, Germany \\ ${ }^{3}$ Beckman Institute, Department of Electrical Engineering and Department of Physics, University of Illinois, Urbana, Illinois 61801, USA
}

(Received 29 March 2011; published 4 January 2012)

\begin{abstract}
It is demonstrated that the three-slit interference, as obtained from explicit solutions of Maxwell's equations for realistic models of three-slit devices, including an idealized version of the three-slit device used in a recent three-slit experiment with light [U. Sinha et al., Science 329, 418 (2010)], is nonzero. The hypothesis that the three-slit interference should be zero is the result of dropping the one-to-one correspondence between the symbols in the mathematical theory and the different experimental configurations, opening the route to conclusions that cannot be derived from the theory proper. It is also shown that under certain experimental conditions, this hypothesis is a good approximation.
\end{abstract}

DOI: 10.1103/PhysRevA.85.012101

PACS number(s): 03.65.Ta, 42.25.Hz, 03.50.De, 42.50.Xa

\section{INTRODUCTION}

According to the working hypothesis (WH) of Refs. [1,2], quantum interference between many different pathways is simply the sum of the effects from all pairs of pathways. In particular, application of the WH to a three-slit experiment yields [2]

$$
I(\mathbf{r}, O O O)=\left|\psi_{1}(\mathbf{r})+\psi_{2}(\mathbf{r})+\psi_{3}(\mathbf{r})\right|^{2},
$$

where $\psi_{j}$, with $j=1,2,3$, represents the amplitude of the wave emanating from the $j$ th slit with the other two slits closed and $\mathbf{r}$ denotes the position in space. Here and in the following we denote the intensity of light recorded in a threeslit experiment by $I(\mathbf{r}, O O O)$, the triple $O$ 's indicating that all three slits are open. We write $I(\mathbf{r}, C O O)$ for the intensity of light recorded in the experiment in which the first slit is closed and so on.

Assuming the WH to be correct, it follows that

$$
\begin{aligned}
I(\mathbf{r}, O O O)= & \left|\psi_{1}(\mathbf{r})+\psi_{2}(\mathbf{r})+\psi_{3}(\mathbf{r})\right|^{2} \\
= & \left|\psi_{1}(\mathbf{r})+\psi_{2}(\mathbf{r})\right|^{2}+\left|\psi_{1}(\mathbf{r})+\psi_{3}(\mathbf{r})\right|^{2} \\
& +\left|\psi_{2}(\mathbf{r})+\psi_{3}(\mathbf{r})\right|^{2}-\left|\psi_{1}(\mathbf{r})\right|^{2} \\
& -\left|\psi_{2}(\mathbf{r})\right|^{2}-\left|\psi_{3}(\mathbf{r})\right|^{2} \\
= & I(\mathbf{r}, O O C)+I(\mathbf{r}, O C O)+I(\mathbf{r}, C O O) \\
& -I(\mathbf{r}, O C C)-I(\mathbf{r}, C O C)-I(\mathbf{r}, C C O) .
\end{aligned}
$$

In other words, still assuming the WH to be correct, we must have

$$
\begin{aligned}
\Delta(\mathbf{r})= & I(\mathbf{r}, O O O)-I(\mathbf{r}, O O C)-I(\mathbf{r}, O C O)-I(\mathbf{r}, C O O) \\
& +I(\mathbf{r}, O C C)+I(\mathbf{r}, C O C)+I(\mathbf{r}, C C O)=0 .
\end{aligned}
$$

In analogy to the expression for the two-slit interference term $I(\mathbf{r}, O O)-I(\mathbf{r}, O C)-I(\mathbf{r}, C O)$ in a two-slit experiment, we refer to $\Delta(\mathbf{r})$ as the three-slit interference term.

\footnotetext{
*h.a.de.raedt@rug.nl

${ }^{\dagger}$ k.michielsen@fz-juelich.de

${ }_{\ddagger}^{\ddagger} \mathrm{k}$-hess@illinois.edu
}

According to Refs. [1,2], the identity in Eq. (3) follows from quantum theory and the assumption that the Born rule $I(\mathbf{r}) \propto|\Psi(\mathbf{r})|^{2}$ holds. In a recent three-slit experiment with light [2], seven contributions to $\Delta(\mathbf{r})$ were measured and taking into account the uncertainties intrinsic to these experiments, it was found that $\Delta(\mathbf{r}) \approx 0$. This finding was then taken as experimental evidence that the Born rule $I(\mathbf{r}) \propto|\Psi(\mathbf{r})|^{2}$ is not violated [2].

The purpose of the present paper is to draw attention to the fact that within Maxwell's theory or quantum theory, the premise that Eq. (1) [which implies Eq. (3)] holds is false. By explicit solution of the Maxwell equations for several three-slit devices, including an idealized version of the three-slit device used in experiment [2], we show that $\Delta(\mathbf{r})$ is nonzero. We also point out that summing up the seven contributions to $\Delta(\mathbf{r})$, which are the outcomes of seven experiments with different slit configurations, requires dropping the one-to-one correspondence between the symbols in the mathematical theory and the different experimental configurations, not satisfying one of the basic criteria of a proper mathematical description of a collection of experiments. However, as we also show, under certain experimental conditions, Eq. (3) might be a good approximation. We present a quantitative analysis of the approximative character of the WH in Eq. (1) and discuss its limitations.

\section{SOLUTION OF MAXWELL'S EQUATION}

The approximative character of the WH in Eq. (1) can be demonstrated by simply solving the Maxwell equations for a three-slit device in which slits can be opened or closed (simulation results for the device employed in the experiment reported in Ref. [2] are presented in Sec. IV). For simplicity, we assume translational invariance in the direction along the long axis of the slits, effectively reducing the dimension of the computational problem by one.

\section{A. Computer simulation of a three-slit device}

In Fig. 1 we show the stationary-state solution of the Maxwell equations, as obtained from a finite-difference timedomain (FDTD) simulation [3] for a three-slit device, with 


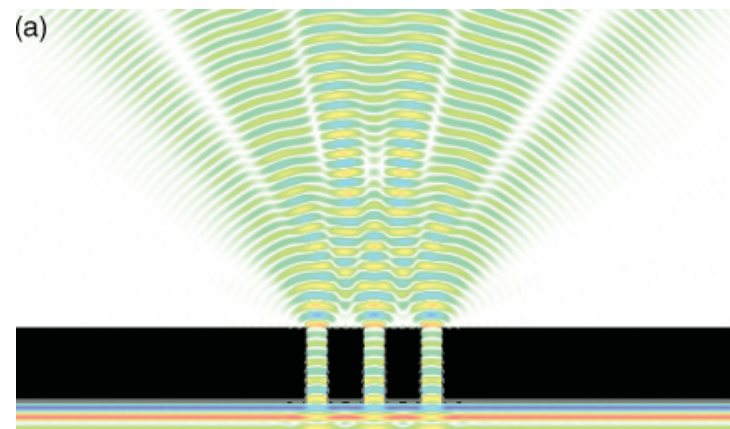

(b)

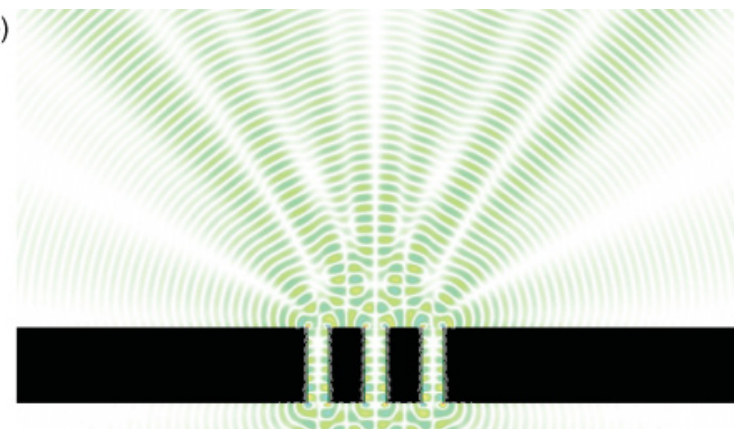

FIG. 1. (Color online) Amplitudes of the (a) $E_{x}$ and (b) $E_{z}$ components of the electric fields as obtained from a FDTD solution of Maxwell's equation for light incident on a metallic plate with three slits. The incident wave is monochromatic and has a wavelength of $\lambda=500 \mathrm{~nm}$. The slits are $\lambda$ wide, their centers being separated by $3 \lambda$. The index of refraction of the $4 \lambda$-thick metallic plate (colored black) is $2.29+2.61 i$. In the FDTD simulations, the material (steel) is represented by a Drude model [3].

slits being $\lambda$ wide and their centers being separated by $3 \lambda$, illuminated by a monochromatic wave with wavelength $\lambda$. From the simulation data, we extract the angular distribution $I(\theta, O O O)$. Repeating these simulations with one and two of the slits closed, we obtain $I(\theta, C O O)$ and so on. In all these simulations, the number of mesh points per wavelength $\lambda$ was taken to be 100 to ensure that the discretization errors of the electromagnetic (EM) fields and geometry are negligible. The simulation box is $75 \lambda \times 40 \lambda$ large (corresponding to
30011501 grid points), terminated by uniaxial perfectly matched layer (UPML) boundaries to suppress reflection from the boundaries [3]. The device is illuminated from the bottom (Fig. 1), using a current source that generates a monochromatic plane wave that propagates in the vertical direction.

In Fig. 2(a) we show a comparison between the angular distribution of the transmitted intensity $I(\theta ; O O O)$ as obtained from the FDTD simulation (solid circles) and Fraunhofer theory (solid line). Plotting

$$
\Sigma(\theta)=\frac{I(\theta ; O O O)-I(\theta ; C O O)-I(\theta ; O C O)-I(\theta ; O O C)+I(\theta ; C C O)+I(\theta ; C O C)+I(\theta ; O C C)}{I(\theta=0 ; O O O)}
$$

as a function of $\theta$ [see Fig. 2(b)] clearly shows that the WH in Eq. (1) of Refs. [1,2] is in conflict with Maxwell's theory: $\Sigma(\theta)$ takes values in the $0.5 \%$ range, which is much too large to be dismissed as numerical noise. Note that $\Sigma(\theta)$ is obtained from data produced by seven different device configurations.

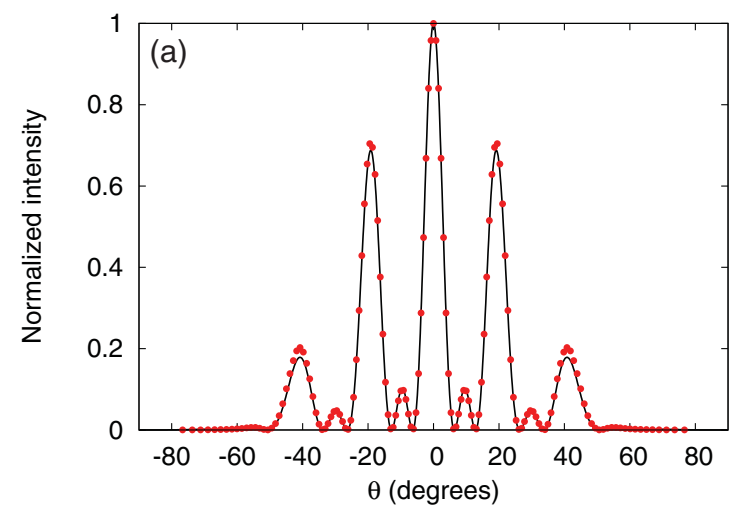

Physically, the fact that $\Sigma(\theta) \neq 0$ is related to the presence of a wave amplitude in the vicinity of the surfaces of the scattering object (one-, two-, or three-slit system) [see, for instance, Fig. 1(b)]. These amplitudes are very sensitive to changes in the geometry of the device, in particular to the presence or absence of a sharp edge. Although these

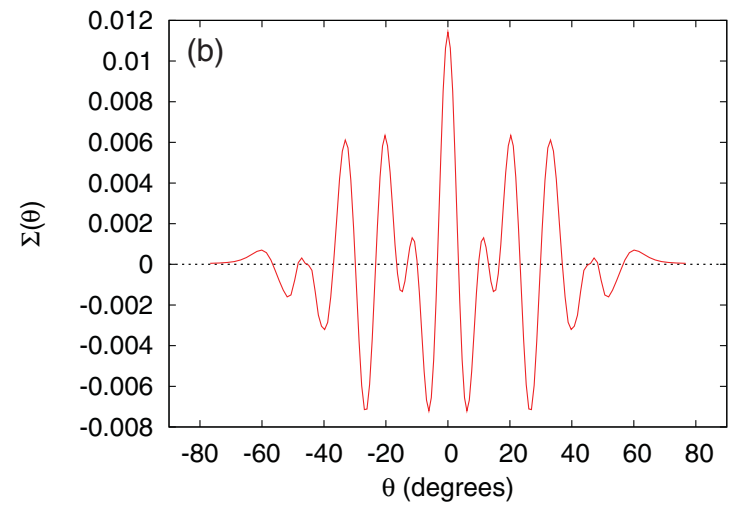

FIG. 2. (Color online) (a) Normalized angular distribution of light $I(\theta ; O O O) / I(\theta=0 ; O O O)$ transmitted by $N=3$ slits (see Fig. 1$)$ as obtained from the FDTD simulation (solid circles) and Fraunhofer theory (solid line) $I(\theta, s=1, d=2, N=3$ ) [see Eq. (6)] where $s$ and $d$ are the dimensionless slit width and slit separation, respectively [4]. (b) Normalized difference $\Sigma(\theta)=[I(\theta ; O O O)-I(\theta ; C O O)-$ $I(\theta ; O C O)-I(\theta ; O O C)+I(\theta ; C C O)+I(\theta ; C O C)+I(\theta ; O C C)] / I(\theta=0 ; O O O)$ as a function of $\theta$. According to the WH of Refs. [1,2], this difference should be zero. 
amplitudes themselves do not significantly contribute to the transmitted light in the forward direction, it is well known that their existence affects the transmission properties of the device as a whole $[5,6]$.

\section{B. Wave decomposition}

The essence of a wave theory is that the whole system is described by only one wave function. Decomposing this wave function in various parts that are solutions of other problems and/or to attach physical relevance to parts of the wave is a potential source for incorrect conclusions and paradoxes. Even for the same problem, the idea to think in terms of waves made up of other waves can lead to nonsensical conclusions, such as that part of a light pulse can travel at a superluminal velocity. Of course, we may express the wave field as a superposition of a complete set of basis functions, e.g., by Fourier decomposition, and this may be very useful in actually solving the mathematical problem (to a good approximation). However, such decompositions are primarily convenient mathematical tricks that, in view of the fact that, in principle, any complete set of basis functions could be used, should not be overinterpreted as being physically relevant $[7,8]$.

The WH in Eq. (1) takes these ideas substantially further by decomposing the wave amplitude into three parts, each part describing the same system (a single slit) located at a different position in space. It is then conjectured that the wave amplitude for the whole system (three slits) is just the sum of these three different amplitudes. Advocates of the physical motivation for this conjecture might appeal to Feynman's path-integral formulation [9] of wave mechanics to justify their picture, but one can see immediately from Feynman's path-integral formalism that the WH in Eq. (1) is in fact not valid.

We use the expression for the propagator of the electron as given by Feynman and assume that the particle proceeds from a location $a$ and time $t_{a}$ on one side of the screen with slits labeled 1,2 , and 3 to a location $b$ where a measurement is taken at time $t_{b}$ on the other side. We assume that there exists some time $t_{c}$ between $t_{a}$ and $t_{b}$ (as assumed by Feynman on p. 36 of Ref. [9]). The propagator for this process is denoted by Feynman as $K(b, a)$ [9]. If we include for clarity the times, then we would have to write $K\left(\left(b, t_{b}\right),\left(a, t_{a}\right)\right)$. As pointed out by Feynman (Ref. [9], p. 57) we have a connection of this propagator to the wave function $\psi$ given by

$$
\psi\left(b, t_{b}\right)=K\left(\left(b, t_{b}\right),\left(a, t_{a}\right)\right)
$$

Feynman represented the propagator $K$ by a path integral that sums over all possible space-time paths to go from $a$ to $b$ with the end-point times as given above. If we have an infinitely extended screen in between $a$ and $b$ with only slit 1 open, then all paths can only proceed through this one slit. We denote the wave function that is calculated for a path leading through a particular point $x_{1}$ of the slit at time $t_{x_{1}}$ by $\psi_{1}^{\prime}$. Similarly for slits 2 and 3 open only we have $\psi_{2}^{\prime}$ and $\psi_{3}^{\prime}$, respectively, and the corresponding $K$ 's are calculated with Feynman paths that only go through slit 2 or 3 , respectively.
Had we chosen all three slits open, then Feynman's formalism insists that pathways going through multiple slits matter in general. Therefore, we would have to include paths through multiple slits in the path-integral representation of $K$ and we would obtain a corresponding $\psi_{123}^{\prime}$. Thus, Feynman's quantum mechanics with all three slits open does contain an infinity of paths that go through multiple slits resulting in $\psi_{123}^{\prime}$. However, none of the wave functions $\psi_{1}^{\prime}, \psi_{2}^{\prime}$, or $\psi_{3}^{\prime}$ may contain any path through more than one slit because of the assumption that only one slit is open at a time. Therefore, all the expressions involving these amplitudes do not contain multiple-slit path integrals and consequently do not contain all the paths that are required to compute $\psi_{123}^{\prime}$. In the following section we illustrate the importance of all by solving the Maxwell equations for a minor variation of the three-slit experiment in which we block one slit.

\section{Three-slit device with blocked middle slit}

The geometry of the device that we consider is depicted in Fig. 3 together with the FDTD solution of the EM fields in the stationary state. We have taken the three-slit device used in Fig. 2 and blocked the middle slit by filling half of this slit with material (the same as that used for other parts of the three-slit device), once from the top [Figs. 3(a) and 3(b)] and once from the bottom [Figs. 3(b) and 3(c)]. Comparing the FDTD solutions shown in Figs. 3(a) and 3(b) and Figs. 3(c) and $3(\mathrm{~d})$, it is obvious to the eye that the wave amplitudes provide no support for the idea that these systems can be described by a wave going through one slit and another wave going through the other slit. The angular distributions for the two cases look very similar [see Fig. 3(e)], but differ on the $1 \%$ level [see Fig. 3(f)].

\section{THE WORKING HYPOTHESIS AS AN APPROXIMATION}

Having presented examples that clearly demonstrate that the WH in Eq. (1) does not hold in general, it is of interest to scrutinize the situations for which the WH in Eq. (1) is a good approximation [10,11]. As pointed out earlier, in general, interference between many different pathways is not simply the sum of the effects from all pairs of pathways. To establish nontrivial conditions under which it truly is a pairwise sum, we discard experiments for which the WH trivially holds, that is, we discard experiments that exactly probe the interference of three waves, such as the extended MachZehnder interferometer experiment described in Ref. [12] and the class of statistical problems described by trichotomous variables considered in Ref. [13].

Let us (1) neglect the vector character of EM waves and (2) assume that the diffraction of the three-slit system is described by Fraunhofer diffraction theory. Then, for normal incidence, the angular distribution of light intensity produced by diffraction from $N$ slits is given by [4]

$$
I(\theta, s, d, N)=\left(\frac{\sin (N \pi d \sin \theta)}{\sin (\pi d \sin \theta)}\right)^{2}\left(\frac{\sin (\pi s \sin \theta)}{\pi s \sin \theta}\right)^{2}
$$




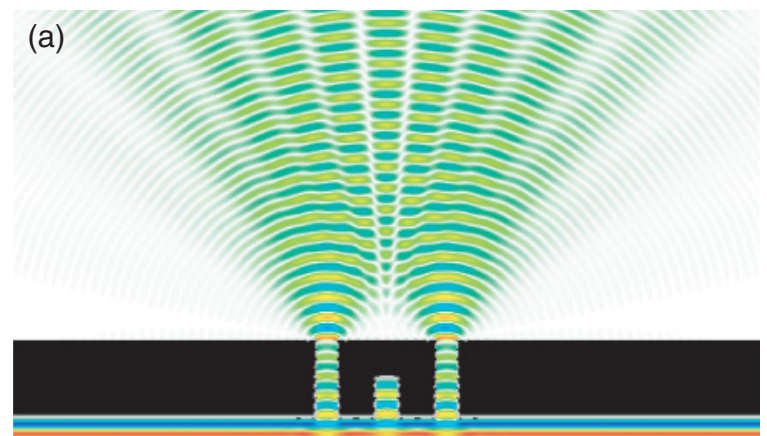

(c)

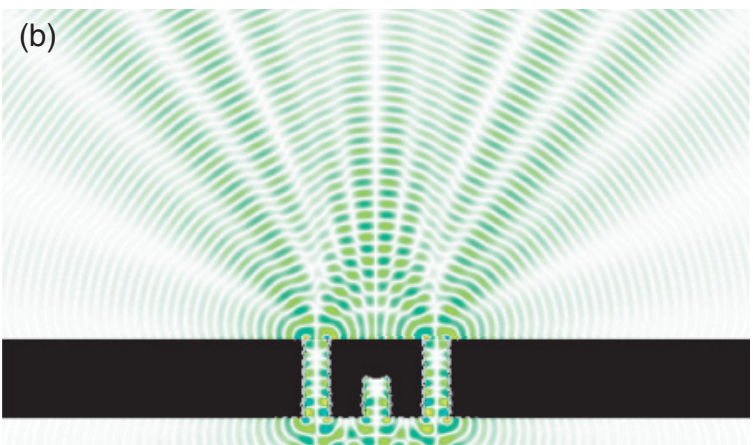

(d)
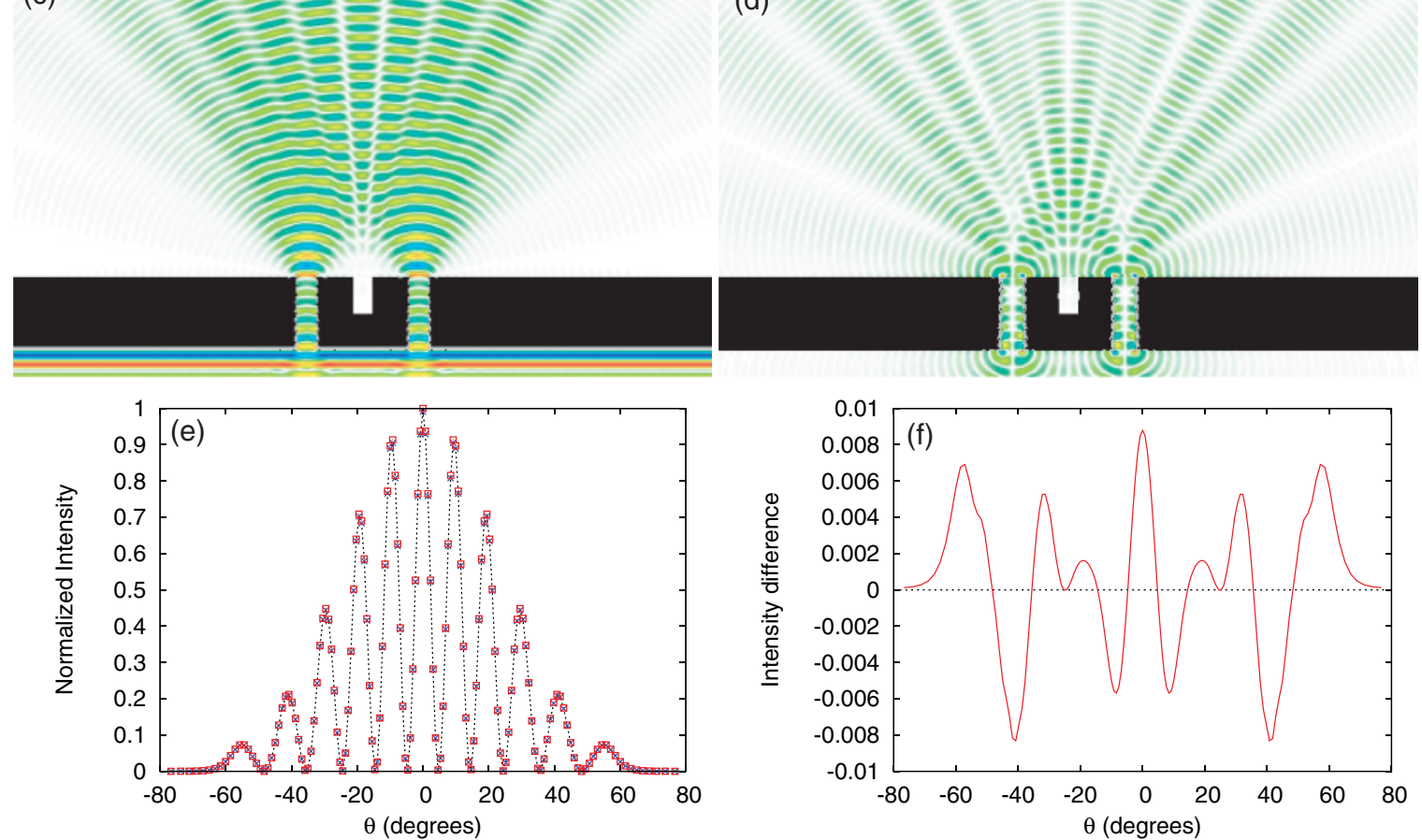

FIG. 3. (Color online) Amplitudes of the (a) and (c) $E_{x}$ and (b) and (d) $E_{z}$ components of the electric fields as obtained from a FDTD solution of Maxwell's equation for light incident on a metallic plate with two slits and a hole between the two slits. The incident wave is monochromatic and has a wavelength of $\lambda=500 \mathrm{~nm}$. The slits are $\lambda$ wide, their centers being separated by $3 \lambda$. The index of refraction of the $4 \lambda$-thick metallic plate (steel, colored black) is $2.29+2.61 i$. The holes are $\lambda$ wide and $2 \lambda$ deep. (e) Normalized angular distribution of light transmitted by the devices extracted from the FDTD simulation data. The closed circles denote simulation results for $I(\theta) / I(\theta=0)$ for the slit in the center filled with material half-way from the bottom [see (c) and (d)], the crosses denote simulation results for $I^{\prime}(\theta) / I^{\prime}(\theta=0)$ for the slit in the center filled with material halfway from the top [see (a) and (b)], and the dashed line is a guide to the eye. On the scale used, the two angular distributions cannot be distinguished. (f) Normalized difference between the angular distributions of the device with the hole in the bottom [(a) and (b)] and the top [(c) and (d)]: $\left[I(\theta)-I^{\prime}(\theta)\right] / \max \left[I(\theta=0), I^{\prime}(\theta=0)\right]$. According to the WH of Refs. [1,2], this difference should be zero.

where $s$ and $d$ are the dimensionless slit width and slit separation expressed in units of the wavelength $\lambda$, respectively. Therefore, we have

$$
\begin{aligned}
\Delta(\theta)= & I(\theta, s, d, 3)-2 I(\theta, s, d, 2)-I(\theta, s, 2 d, 2) \\
& +3 I(\theta, s, d, 1) \\
= & {\left[(1+2 \cos 2 a d)^{2}-8 \cos ^{2} a d-4 \cos ^{2} 2 a d+3\right] } \\
& \times\left(\frac{\sin a s}{a s}\right)^{2}=0
\end{aligned}
$$

where $a=\pi \sin \theta$. Thus, in the Fraunhofer regime the WH in Eq. (1) holds.
It is not difficult to see that $\Delta(\theta)=0$ is an accident rather than a general result by simply writing down the Maxwell curl equations $[3,4]$

$$
\begin{aligned}
& \varepsilon(\mathbf{r}) \frac{\partial \mathbf{E}(\mathbf{r}, t)}{\partial t}=\nabla \times \mathbf{H}(\mathbf{r}, t)-J(\mathbf{r}, t), \\
& \mu(\mathbf{r}) \frac{\partial \mathbf{H}(\mathbf{r}, t)}{\partial t}=\nabla \times \mathbf{E}(\mathbf{r}, t)
\end{aligned}
$$

where the geometry of the device is accounted for by the permittivity $\varepsilon(\mathbf{r})$ and, for simplicity, as is often done in optics [4], we may assume that the permeability $\mu(\mathbf{r})=1$.

Let us write $\varepsilon(\mathbf{r}, O O O)$ for the permittivity of the three-slit geometry and $\mathbf{E}(\mathbf{r}, t, O O O)$ and $\mathbf{H}(\mathbf{r}, t, O O O)$ for the 

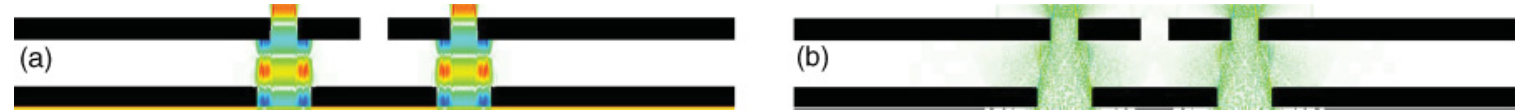

FIG. 4. (Color online) Two-dimensional representation of the experiment reported in Ref. [2]. The three slits at the top are $30 \mu \mathrm{m}$ wide, their centers being separated by $100 \mu \mathrm{m}$. The blocking mask at the bottom can have one, two, or three slits, each slit being $60 \mu \mathrm{m}$ wide with its center aligned with one of the slits in the top plate [2]. In the example shown, the middle slit of the blocking mask is closed (corresponding to the case $O C O$ ). The separation between the top plate and blocking mask is $50 \mu \mathrm{m}$. The index of refraction of the $25-\mu \mathrm{m}$-thick material (colored black) is $2.29+2.61 i$ (index of refraction of steel at $405 \mathrm{~nm}$ ). The wavelength of the incident light is $405 \mathrm{~nm}$. Also shown are the amplitudes of the (a) $E_{x}$ and (b) $E_{z}$ components of the electric fields as obtained from a FDTD solution of Maxwell's equation for a monochromatic light source (not shown) illuminating the blocking mask. Note that the $E_{x}$ and $E_{z}$ components propagate in a very different manner.

corresponding solution of the Maxwell equations [Eqs. (8)]. The WH in Eq. (1) asserts that there should be a relation between $(\mathbf{E}(\mathbf{r}, t, O O O), \mathbf{H}(\mathbf{r}, t, O O O))$ and $(\mathbf{E}(\mathbf{r}, t, C O O)$, $\mathbf{H}(\mathbf{r}, t, C O O)), \quad(\mathbf{E}(\mathbf{r}, t, O C O), \quad \mathbf{H}(\mathbf{r}, t, O C O)), \ldots$, $(\mathbf{E}(\mathbf{r}, t, O C C), \mathbf{H}(\mathbf{r}, t, O C C))$, but this assertion is absurd: There is no theorem in Maxwell's theory that relates the solutions for the case $\varepsilon(\mathbf{r}, O O O)$ to solutions for the cases $\varepsilon(\mathbf{r}, C O O), \ldots, \varepsilon(\mathbf{r}, O C C)$. The Maxwell equations are linear equations with respect to the EM fields, but solutions for different $\varepsilon$ 's cannot simply be added.

Of course, this general argument applies to the Schrödinger equation as well. For a particle moving in a potential, we have

$$
i \hbar \frac{\partial \Psi(\mathbf{r}, t)}{\partial t}=\left(\frac{1}{m} \mathbf{p}^{2}+V(\mathbf{r})\right) \Psi(\mathbf{r}, t)
$$

In essence, the WH in Eq. (1) asserts that there is a relation between the solutions of four problems defined by the potential $V(\mathbf{r})$ and three other potentials $V_{j}(\mathbf{r})$ for $j=1,2,3$. More specifically, it asserts that

$$
\Psi(\mathbf{r}, t)=\Psi_{1}(\mathbf{r}, t)+\Psi_{2}(\mathbf{r}, t)+\Psi_{3}(\mathbf{r}, t)
$$

and

$$
\begin{aligned}
V(\mathbf{r}) \Psi(\mathbf{r}, t) & =V(\mathbf{r}) \Psi_{1}(\mathbf{r}, t)+V(\mathbf{r}) \Psi_{2}(\mathbf{r}, t)+V(\mathbf{r}) \Psi_{3}(\mathbf{r}, t) \\
& =V_{1}(\mathbf{r}) \Psi_{1}(\mathbf{r}, t)+V_{2}(\mathbf{r}) \Psi_{2}(\mathbf{r}, t)+V_{3}(\mathbf{r}) \Psi_{3}(\mathbf{r}, t) .
\end{aligned}
$$

The authors could not think of a general physical situation that would result in Eq. (11).

In summary, $\Delta(\theta) \neq 0$ in experiments not exactly probing the interference of three waves, but $\Delta(\theta)=0$ in experiments carried out in the Fraunhofer regime. In real laboratory experiments, such as the one reported in Ref. [2], it is very difficult, but not impossible, to measure $\Delta(\theta)=0$. Therefore, in the following section we present a computer simulation study of this experiment resulting in a quantitative analysis of the applicability of the WH in Eq. (1).

\section{COMPUTER SIMULATION OF THE EXPERIMENT REPORTED IN REF. [2]}

The geometry of this device is depicted in Fig. 4 (see also Ref. [2]), together with the stationary-state FDTD solution of the Maxwell equations. In the simulation, the device is illuminated from the bottom (Fig. 1), using a current source that generates a monochromatic plane wave that propagates in the vertical direction. The wavelength of the light, the dimension of the slits and their separation, blocking masks, and material properties are taken from Ref. [2]. In view of the large (compared to wavelength) dimensions of the slits, to reduce the computational burden, we assume translational invariance in the direction along the long axis of the slits. This idealization of the real experiment does not affect the conclusions; on the contrary, it eliminates effects of the finite length of the slits. In all these simulations, the 81 mesh points per wavelength $(\lambda=405 \mathrm{~nm})$ were taken to ensure that the discretization errors of the EM fields and geometry are negligible. The simulation box of $820 \times 120 \mu \mathrm{m}^{2}$ (corresponding to 3936188 001 grid points) contains UPMLs to eliminate reflection from the boundaries [3]. Each calculation requires about $900 \mathrm{~GB}$ of memory and took about $12 \mathrm{~h}$, using 8192 processors of the IBM BlueGene/P at the Jülich Supercomputing Centre.

Qualitatively, Fig. 4(a) indicates that the component $E_{x}$ of the EM field propagates through the two layers of slits with very little diffraction from the top (blocking) layer. This is not the case for the $z$ component shown in Fig. 4(b). In this idealized simulation setup, the amplitude of the $y$ component of the EM field is zero.

In Fig. 5(a) we present the results for the angular distribution of the seven cases $(O O O, O O C, O C O, C O O, O C C, C O C$, and $C C O$ ), extracted from seven FDTD simulations. From Fig. 5(b), it is clear that $\kappa(\theta)$, defined as [2]

$$
\kappa(\theta)=\frac{I(\theta ; O O O)-I(\theta ; C O O)-I(\theta ; O C O)-I(\theta ; O O C)+I(\theta ; C C O)+I(\theta ; C O C)+I(\theta ; O C C)}{|I(\theta ; O O C)-I(\theta ; O C C)-I(\theta ; C O C)|+|I(\theta ; C O O)-I(\theta ; C O C)-I(\theta ; C C O)|+|I(\theta ; O C O)-I(\theta ; O C C)-I(\theta ; C C O)|},
$$

is not identically zero, but of the order of $10^{-5}$. Note that Eq. (12) exactly corresponds to the expression for $\kappa$ defined in Ref. [2] since in the idealization of the real experiment $I(\theta ; C C C)=0$. In Ref. [2] it is reported that 

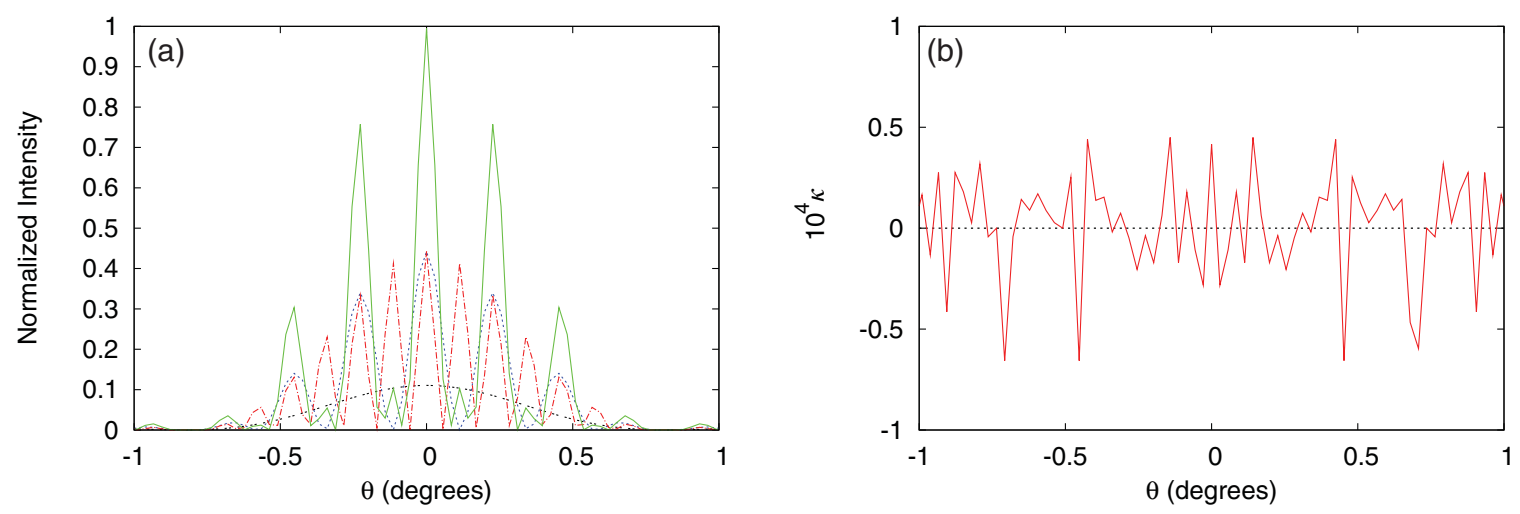

FIG. 5. (Color online) (a) Angular distribution of light transmitted by the system shown in Fig. 4 for the cases in which all slits are open ( $O O O$, solid line), one slit is closed ( $C O O$ and $O O C$, dashed line; $O C O$, dash-dotted line), and two slits are closed $(C C O, C O C$, and $O C C$, double-dotted line), as obtained from FDTD simulations. (b) $\kappa$ as a function of $\theta$, as defined by Eq. (12). According to the WH [1,2], this difference should be zero.

$\kappa(\theta=0)=0.0064 \pm 0.0120$ for measurements with single photons, $\kappa(\theta=0)=0.0073 \pm 0.0018$ for measurements with a laser source and a power meter for detection, and $\kappa(\theta=$ $0)=0.0034 \pm 0.0038$ for measurements with a laser source attenuated to single-photon level and a silicon avalanche photodiode for detection. The upper bound for $\kappa$ at several detector positions given by the experiment [2] is $\kappa(\theta)<10^{-2}$. We find that for the idealized version of the experiment $\kappa(\theta=0)=4 \times 10^{-5}$ and $\kappa(\theta)<7 \times 10^{-5}$, which are a factor 100 smaller than the values measured in the experiment. Note that the experimental and simulated values for $\kappa$ are very small because the experiment is carried out in a regime in which scalar Fraunhofer theory works well, as can be expected from the dimensions of the slits and slit separations of the device.

\section{DISCUSSION}

A necessary condition for a mathematical model to give a logically consistent description of the experimental facts is that there is one-to-one correspondence between the symbols in the mathematical description and the actual experimental configurations. When applied to the three-slit experiment in which one slit or two slits may be closed, the argument that leads from Eq. (1) to Eq. (2) is false because there is no such correspondence.

If $\psi_{j}$ in Eq. (1) is to represent the amplitude of the wave emanating from the $j$ th slit with all other slits closed, the WH should be written as

$$
I(\mathbf{r}, O O O)=|\psi(\mathbf{r}, O C C)+\psi(\mathbf{r}, C O C)+\psi(\mathbf{r}, C C O)|^{2},
$$

that is, we should label the $\psi$ 's such that there can be no doubt about the experiment that they describe. This notation establishes the necessary one-to-one correspondence between the mathematical description (the $\psi$ 's) of the particular experiment (labeled by $O C C$, etc.). Now we have

$$
\begin{aligned}
I(\mathbf{r}, O O O)= & |\psi(\mathbf{r}, O C C)+\psi(\mathbf{r}, C O C)|^{2}+\mid \psi(\mathbf{r}, O C C) \\
& +\left.\psi(\mathbf{r}, C C O)\right|^{2}+|\psi(\mathbf{r}, C O C)+\psi(\mathbf{r}, C C O)|^{2}
\end{aligned}
$$

$$
\begin{aligned}
& -|\psi(\mathbf{r}, O C C)|^{2}-|\psi(\mathbf{r}, C C O)|^{2} \\
& -|\psi(\mathbf{r}, C C O)|^{2}
\end{aligned}
$$

At this point, it is simply impossible to bring Eq. (14) into the form of Eq. (2) without making the assumption that

$$
\begin{aligned}
& \psi(\mathbf{r}, O O C)=\psi(\mathbf{r}, O C C)+\psi(\mathbf{r}, C O C), \\
& \psi(\mathbf{r}, O C O)=\psi(\mathbf{r}, O C C)+\psi(\mathbf{r}, C C O), \\
& \psi(\mathbf{r}, C O O)=\psi(\mathbf{r}, C O C)+\psi(\mathbf{r}, C C O) .
\end{aligned}
$$

If we accept this assumption, we recover Eq. (2). However, the assumption expressed by Eq. (15) cannot be justified from general principles of quantum theory or Maxwell's theory: The only way to justify Eq. (15) is to forget that the $\psi$ 's are labeled by the type of experiment (e.g., $O C C$ ) they describe. For a discussion of this point in the case of a two-slit experiment, see Refs. [14,15].

In other words, accepting Eq. (15) destroys the one-toone correspondence between the symbols in the mathematical theory and the different experimental configurations, opening the route to conclusions that cannot be derived from the theory proper. Hence, if $\Delta(\mathbf{r}) \neq 0$ for a three-slit experiment, one cannot conclude that Born's rule does not strictly hold.

From the preceding it is clear that, in general (excluding special cases such as the Fraunhofer regime), the three-slit interference pattern cannot be obtained using a combination of single-slit and two-slit devices. In order to measure the three-slit interference pattern, a three-slit device with all three slits open is required. Similarly, for the measurement of the two-slit interference pattern a two-slit device is required; a combination of measurements using two single-slit devices is not sufficient. A similar issue was raised in Ref. [16] concerning the measurement of arbitrary Hermitian operators acting on the Hilbert space of a spin- $S$ particle using suitable generalized Stern-Gerlach apparatuses. The standard SternGerlach apparatus with an inhomogeneous magnetic field whose direction is constant, but whose magnitude depends on the position, can measure any Hermitian operator of a spin-1/2 system. This is not the case, however, for spin- $S$ systems with $S>1 / 2$ [16]. In order to measure all spin- $S$ 
operators a generalized Stern-Gerlach apparatus, using both electric and magnetic fields, is required [16].

\section{CONCLUSION}

The results of this paper can be summarized as follows.

The three-slit interference, as obtained from explicit solutions of Maxwell's equations for realistic models of three-slit devices, is nonzero.

The hypothesis [1,2] that the three-slit interference in Eq. (3) is zero is false because it requires dropping the one-toone correspondence between the symbols in the mathematical theory and the different experimental configurations opening a route to conclusions that cannot be derived from the theory proper.

Although not holding in general, the hypothesis that the three-slit interference in Eq. (3) should be zero is a good approximation in experiments carried out in the Fraunhofer regime. The experiment reported in Ref. [2] is carried out in this regime and bounds the magnitude of the three-slit interference term to less than $10^{-2}$ of the expected two-slit interference at several detector positions [2]. By explicit solution of the Maxwell equations for an idealized version of the three-slit experiment used in Ref. [2] we provide a quantitative analysis of the approximative character of the hypothesis that the three-slit interference in Eq. (3) is zero. We find that the magnitude of the three-slit interference term is several orders of magnitude smaller than the upper bound found in the experiment [2].

\section{ACKNOWLEDGMENTS}

This work was partially supported by National Computing Facilities, the Netherlands. Part of the calculations has been performed on JUGENE at JSC under VSR project 4331.
[1] R. D. Sorkin, Mod. Phys. Lett. 9, 3119 (1994).

[2] U. Sinha, C. Couteau, T. Jennewein, R. Laflamme, and G. Weihs, Science 329, 418 (2010).

[3] A. Taflove and S. Hagness, Computational Electrodynamics: The Finite-Difference Time-Domain Method (Artech House, Boston, 2005).

[4] M. Born and E. Wolf, Principles of Optics (Pergamon, Oxford, 1964).

[5] G. Gay, O. Alloschery, B. Viaris de Lesegno, C. O'Dwyer, J. Weiner, and H. J. Lezec, Nature Phys. 2, 262 (2006).

[6] P. Lalanne and J. P. Hugonin, Nature Phys. 2, 551 (2006).

[7] C. Roychoudhuri, in Quantum Theory: Reconsideration of Foundations - 5, edited by A. Y. Khrennikov, AIP Conf. Proc. No. 1232 (AIP, New York, 2010), pp. 143-152.
[8] C. Roychoudhuri, J. Nanophoton. 4, 043512 (2010).

[9] R. P. Feynman and A. R. Hibbs, Quantum Mechanics and Path Integrals (McGraw-Hill, New York, 1965).

[10] A. Khrennikov, Phys. Lett. A 372, 6588 (2008).

[11] C. Ududec, H. Barnum, and J. Emerson, Found. Phys. 41, 396 (2011).

[12] J. Franson, Science 329, 396 (2010).

[13] P. Nyman and I. Basieva, in Advances in Quantum Theory, edited by G. Jaeger, A. Khrennikov, M. Schlosshauer, and G. Weihs, AIP Conf. Proc. No. 1327 (AIP, New York, 2011), p. 439.

[14] L. E. Ballentine, Am. J. Phys. 54, 883 (1986).

[15] L. E. Ballentine, Quantum Mechanics: A Modern Development (World Scientific, Singapore, 2003).

[16] A. Swift and R. Wright, J. Math. Phys. 21, 77 (1980). 